\title{
IDENTIFICATION OF THE COMBUSTION ENGINE RESISTANCE TO MOTION TORQUE COMPONENTS
}

Identification of the combustion engine resistance to motion is necessary in all investigations aimed at limiting frictional losses. Despite many attempts to measure the frictional resistance components, there are still no functional test beds that would allow to take measurements of frictional force or frictional torque in particular units. Most often the mean value of the torque for one or even two full revolutions of the crankshaft is determined. For many reasons it is more convenient to determine the resistance to motion torque of the combustion engine with an external drive but unfortunately, the torque from the external drive is disturbed by torsional vibration. There is at least a theoretical possibility of separating the torque generated by vibration from the raw measuring signal. In the paper, the results obtained as well as selected problems of such separation are presented.

Keywords: internal combustion engine, coupling torque, harmonic components, proper vibration of the crankshaft

\section{Introduction}

Identification of the combustion engine resistance to motion is necessary in all investigations aimed at limiting frictional losses. Despite many attempts to measure the frictional resistance components, no functional test beds that would allow to take measurements of frictional force or frictional torque in particular units have been constructed yet. Usually it is the value of the coupling torque of the engine with the power receiver that is the basic measurement during the combustion engine investigations. Most often the mean value of the torque for one or even two full revolutions of the

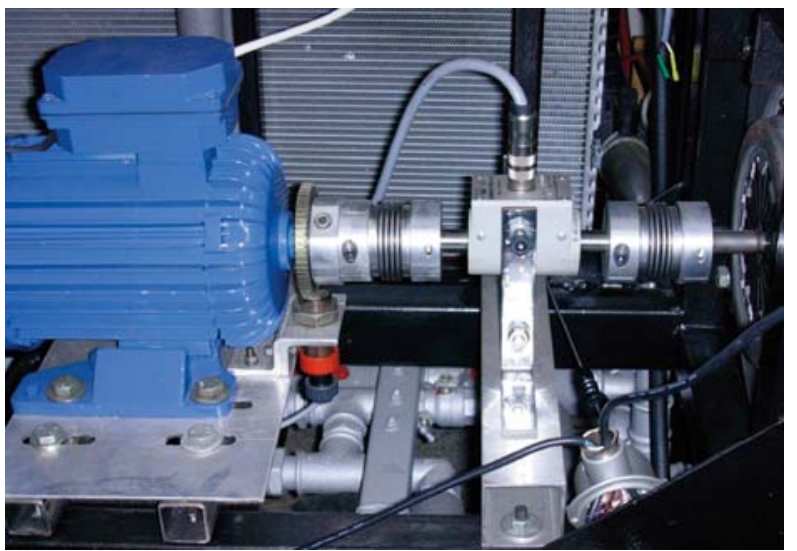

Fig. 1. The test bed of the two-cylinder combustion engine of a passenger car equipped with the measurement set for the instantaneous torque values crankshaft is determined. The test bench equipped with a torque sensor is shown in Fig. 1.

The resistance to motion torque of the combustion engine accompanies rotation of the crankshaft regardless of whether the engine is in normal operation or if it is revolved by the external engine. For many reasons, it is more convenient to determine the resistance to motion torque of the combustion engine with the external drive. Unfortunately, the torque from the external drive is disturbed by torsional vibration because the rotor of the driving engine creates the vibratory unit with the tested engine crankshaft. For this reason, the torque sensor placed between the rotor and the crankshaft measures the torque which is the sum of the resistance to motion torque of the investigated engine and the torque generated by vibration. Because in a longer period of time the frequency of the torque generated by vibration is usually not the exact multiplicity of the driving engine rotational frequency, there is at least a theoretical possibility of separating the torque generated by vibration. In the paper, the results obtained as well as selected problems of such separation are presented.

2. Torque transmitted from the driving engine shaft to the crankshaft of the investigated combustion engine

In Fig. 2, the thinnest blue line presents the course of torque. It was measured on the test bed at $3000 \mathrm{rpm}$.

It can be noticed that the course of the measured signal is not repeatable in the following working cycles. The work character of the piston engine determines the periodicity formation of resis-

\footnotetext{
* Antoni Iskra, Maciej Babiak, Jaroslaw Kaluzny

Institute of Combustion Engines and Transportation, Faculty of Working Machines and Transportation, Poznan University of Technology, Poland, E-mail: Antoni.Iskra@put.poznan.pl
} 


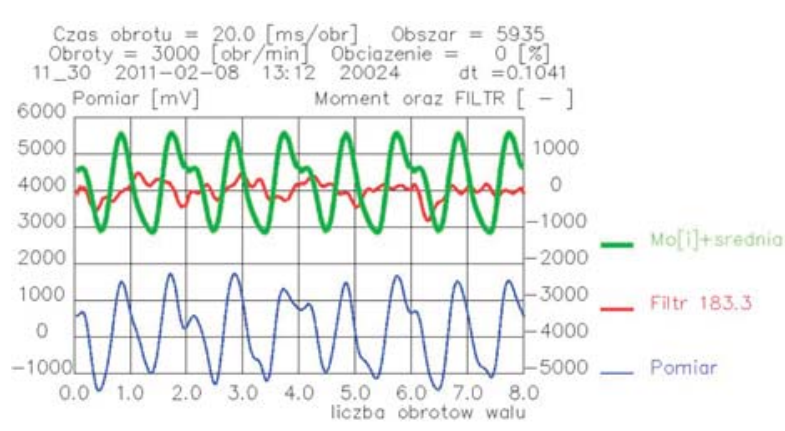

Fig. 2. The course of torque measured with the torque sensor - the thinnest blue line, torque obtained after excluding harmonic components which are the multiplicity of the 720 degrees working cycle - the thickest green line, the sum of harmonic components excluded from the measured signal - red average thickness line. Key: Czas obrotu- revolution time, obszar - area, obroty-revolutions, obciażenie load, pomiar - measurement, Moment oraz filtr - torque and filter, liczba obrotów watu - crankshaft revolutions

tance to motion components. These components are mainly: the resistance of compression, resistance of inertia, frictional resistance. As a result, the coupling torque of the drive with the engine on the test bed shown in Fig. 1 should repeat itself in cycles of two revolutions of the crankshaft. The only component of the coupling torque whose frequency is different from the shaft revolutions frequency is the frequency of various proper vibration forms. Theoretically it is possible that the frequency of one of the forms is close to the shaft rotational speed but this phenomenon is easy to detect.

What results from the described regularity is that the harmonic different from the multiplication of shaft revolutions frequency can be excluded. As a result of performing the described operation, the course of torque was obtained and is pointed out in Fig. 2 with the thickest green line. Additionally, the red line presents the summary course of excluded harmonic components.

\section{Comparison of the revised resistance to move torque with the calculated torque of the investigated engine}

Making the static course - that is excluding the shaft proper vibrations- of the resistance to move torque is a typical operation connected with designing a new engine. Despite omitting the shaft proper vibration, the static course of torque allows to estimate energetic characteristics of the new engine but such course is of little use in strength calculations. However, in order to evaluate the obtained course of the revised resistance to move torque which is shown in Fig. 2, a comparison with the calculated statistic course shown in Fig. 3 was made.

As it can be noticed there are some distinctions between the character of the modified measured course (Fig. 2) and the character of the simulated course (Fig. 3). It mainly concerns the repeatability of courses. The measured signal repeats itself every

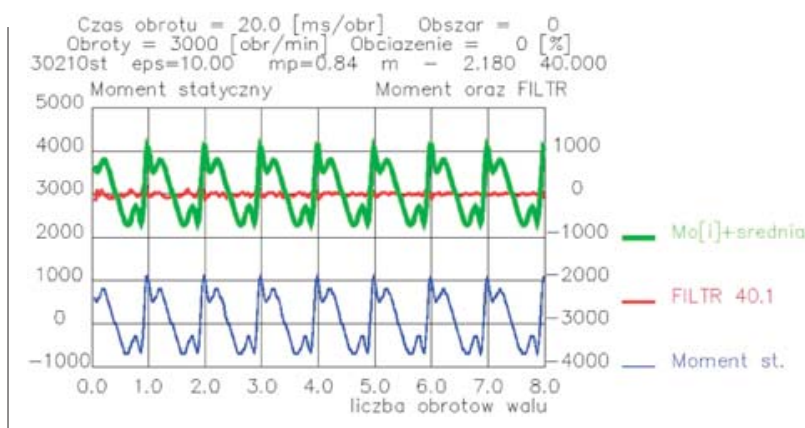

Fig. 3. The course of the static torque obtained with use of a computer simulation - the thinnest blue line, The course of torque obtained after excluding harmonic components which are the multiplicity of the 720 degrees working cycle - the thickest green line, the sum of harmonic components excluded from the measured signal - red average thickness line

two revolutions and the simulated signal recurs every single revolution. Because courses concern the two-cylinder four-stroke engine, the repeatability of static course is obvious. But the results of the revised measurements do not confirm the seemingly obvious computer simulation results. It is likely to result from a greater torsional deflection of the crank more distant from the torque sensor.

\section{The influence of engine speed accuracy on excluding proper vibration components from the measured signal}

The presented method of revising the measured signal of the coupling torque is fraught with many errors. The main problem is the precise determination of the periodical torque components frequencies resulting from the engine function and not from the proper vibration. In order to determine how the engine speed measurement error influences the obtained revised signal, an analysis

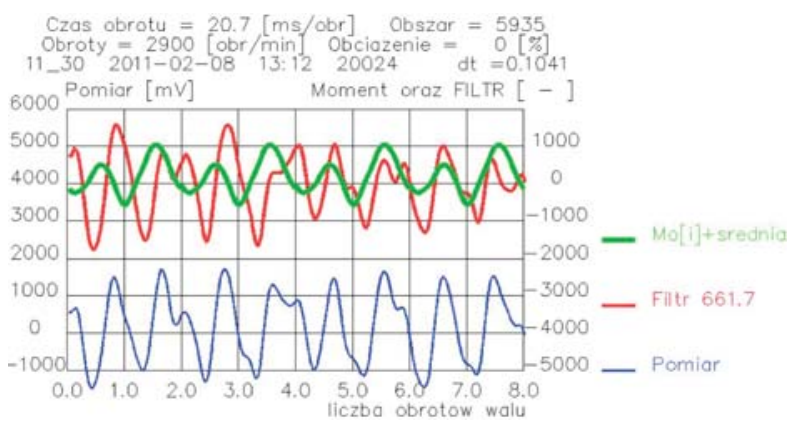

Fig. 4. The torque course from torque sensor - the thinnest blue line, The course of torque obtained after excluding harmonic components which are the multiplicity of the 720 degrees working cycle - the thickest green line, the sum of harmonic components excluded from the measured signal - red average thickness line. The assumed engine speed is $3 \%$ less than the actual engine speed 
was made which was analogous to the one presented in Fig. 2 but with the engine speed incorrectly assumed at $2900 \mathrm{rpm}$ instead of $3000 \mathrm{rpm}$. The obtained revised course is presented in Fig. 4.

It is easy to notice that if the rotational speed is $3 \%$ less than the actual engine speed, the revised course differs from both the actual engine speed revised course (Fig. 2) and the course obtained with use of computer simulations (Fig. 3).

It is only if the actual engine speed dispersion is less than $0.5 \%$ from the assumed for revising that it basically does not influence the correctness of the realized revising (Fig. 5)

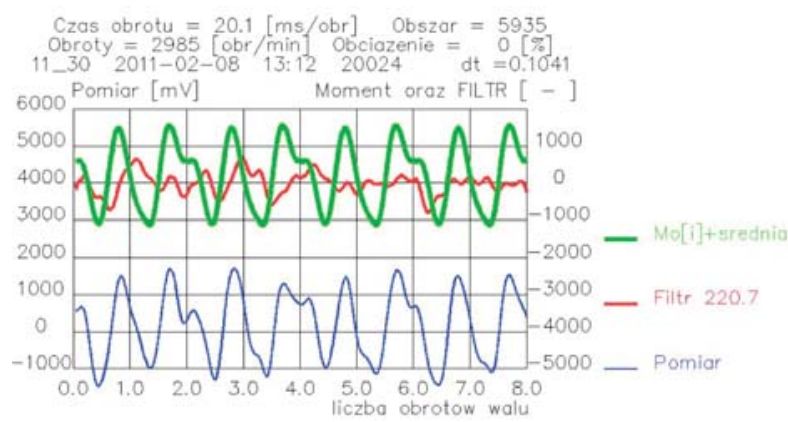

Fig. 5. The torque course from torque sensor - the thinnest blue line, the course of torque obtained after excluding harmonic components which are the multiplicity of the 720 degrees working cycle - the thickest green line, the sum of harmonic components excluded from the measured signal - red average thickness line. The assumed engine speed is $0,5 \%$ less than the actual engine speed

\section{Repeatability of courses}

The results of torque measurements presented earlier are adequate to the conditions in which the component generated by inertia forces has a substantial share and therefore at a high engine shaft speed. In case of lower values of the rotary speed close to the neutral run, there occur components in the signal of the resistance to motion torque which significantly disrupt the anticipated torque course. Apart from two fundamental components (the torque generated by gas and friction forces) there occurs a relatively strong torque generated by vibrations in the combustion engine - electric motor unit. In the case when the vibrations torque component frequency is not the multiplicity of rotations frequency, the applied computer program allows to exclude such a component. However, if the vibrations component frequency is close to the multiplicity of rotations frequency, such a component is difficult to identify since it can be generated by both friction and vibrations. However, the hitherto conducted research shows that usual vibrations generate high frequencies, whereas friction is quite well modeled by low frequencies represented by the harmonic lower than 33rd order for a two-cylinder four-stroke engine. The correctness of the presented thesis is documented by the courses shown in Figs. $6-9$.

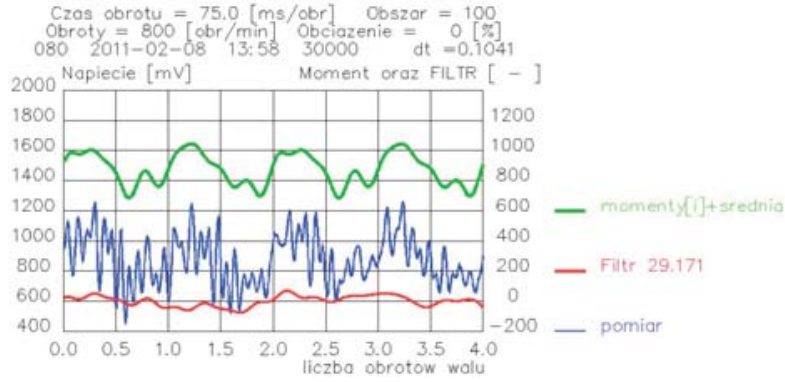

Fig. 6. The course of torque measured with the torque sensor - the thinnest blue line, torque obtained after excluding harmonic components which are the multiplicity of the 720 degrees working cycle - the thickest green line, the sum of harmonic components excluded from the measured signal - red average thickness line; the courses obtained from measurement point $\mathrm{nr} 100$

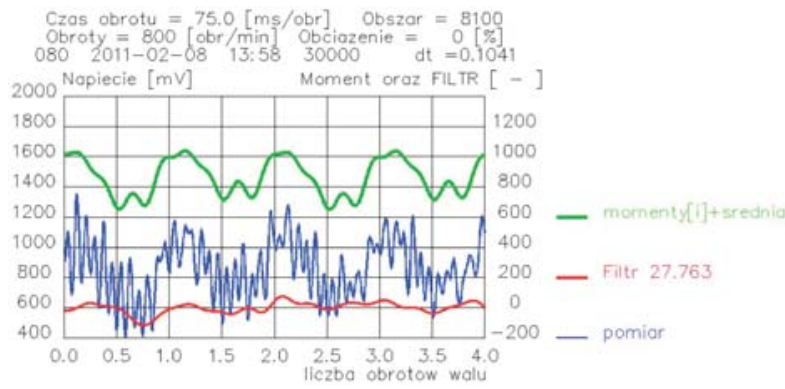

Fig. 7. The course of torque measured with the torque sensor - the thinnest blue line, torque obtained after excluding harmonic components which are the multiplicity of the 720 degrees working cycle - the thickest green line, the sum of harmonic components excluded from the measured signal - red average thickness line; the courses obtained from measurement point $\mathrm{nr} 8100$

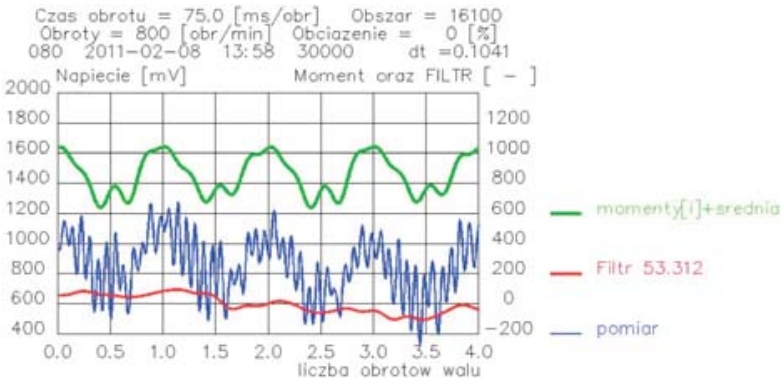

Fig. 8. The course of torque measured with the torque sensor - the thinnest blue line, torque obtained after excluding harmonic components which are the multiplicity of the 720 degrees working cycle - the thickest green line, the sum of harmonic components excluded from the measured signal - red average thickness line; the courses obtained from measurement point $n r 16100$

These figures present the unstable measured signal - the thinnest blue line and the sum of 32 harmonics - the green line. 
In spite of the fact that the course in Fig. 7 was obtained with the delay of 8000 measuring points, and in Fig. 8 - of 16000 points, the obtained revised courses marked green are very similar. The performed reasoning shows that the revised courses mainly result from the influence of gas and friction forces. Since the gas forces result quite accurately from the realization of the thermodynamic cycle, one can easily subtract the torque generated by gas forces from the revised torque and obtain as a result a pure form of the torque generated by friction forces. One should emphasize that the presented reasoning is accurate under the condition of a precise repeatability of the revised torque, which is proven by Figs. 6-8.

In order to compare the obtained research results with the results of the computer simulation, a summary course of the torques generated by gas and inertia forces is presented in Fig. 9 .

Therefore, the differences in the courses in Figs. 8 and 9 result from the omission of friction forces in the simulation.

\section{Conclusion}

The presented method of revising the measured resistance to motion torque of the combustion engine is necessary to determine

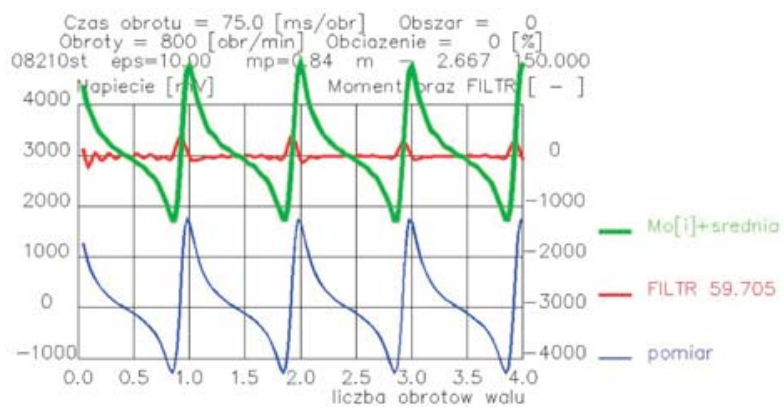

Fig. 9. The course of the static torque obtained in a computer simulation - the thinnest blue line, the course of torque obtained after excluding harmonic components which are the multiplicity of the 720 degrees working cycle - the thickest green line, the sum of harmonic components excluded from the signal of the computer simulation

- red average thickness line; rotational speed - 800 [rpm]

instantaneous torque values, i.e. to evaluate the internal friction components in the combustion engine. This method requires the precise maintaining of a constant engine speed. It is recommended that the engine speed should be specified with the accuracy of not less than $0.5 \%$.

\section{References}

[1] ISKRA, A.: Dynamics of Mechanisms of Combustion Piston Engines (in Polish), Wydawnictwo PP, Poznan 1995. 